\title{
A Mean Field Voltage Control Approach for Active Distribution Networks with Uncertainties
}

\author{
Boyuan Wei, Student Member, IEEE, Zhifeng Qiu, Member, IEEE, and Geert Deconinck, Senior Member, IEEE
}

\begin{abstract}
A model-free distributed control scheme that implements active voltage control in low voltage distribution network (LVDN) is proposed. By solving an individual Hamilton-JacobiBellman-Flemming function with public information, users can compute a good approximation to their optimal control trajectory and take uncertainties into account in a distributed manner. A detailed mathematical framework is given, accompanied by a discussion on the different entries of uncertainties. The proposed control scheme uses a broadcast signal to indicate the probability distribution in mean field theory, and to streamline the demand on Fokker-Planck-Kolmogorov PDE or Mc-Kean Vlasov SDE, which relieves the computational burden. A realistic semiurban distribution network is modified as the study case, with a benchmark of centralized ACOPF to study the performance of the proposed approach. Moreover, two special cases including communication latency and packet loss are given as well, in order to test the robustness of the proposed approach. The results prove that the proposed approach is able to deliver good approximation to the optimal control with uncertainty in a model-free and distributed manner.
\end{abstract}

Index Terms-Active distribution networks, Distributed optimization, Mean field theory, Hamilton-Jacobi-Bellman equation, Model-free, Voltage control.

\section{INTRODUCTION}

W ITH the development of distributed energy resources (DERs), renewable penetration increases not only in medium voltage distribution network, but also low voltage distribution network (LVDN). Along with this trend, conventional passive distribution networks are being transformed into active distribution network (ADN) [1]. Meanwhile, shapeable/deferrable loads spring up in LVDNs, which enlarges the applicability of ADN. Nevertheless, the implementation of $\mathrm{ADN}$ is the synonym of a dramatic increase in the amount of smart meters, sensors, and control devices, via bidirectional communication networks. On one hand, high burdens on both communication and computation makes the infrastructure and operation expensive; on the other hand, the rising demand in data collection is being more and more discordant with the awakening public concerns on the privacy protection of data. In this paper, the term users denotes all the DERs connected to LVDNs, which includes but is not limited to photovoltaics $(\mathrm{PV})$, residential wind turbines, smart households and so on. Therefore, the main challenge to implement active controls

B. Wei and G. Deconinck are with the Department of Electrical Engineering (ESAT), KU Leuven, Leuven, 3001 Belgium. e-mail: Boyuan.Wei@kuleuven.be.

Z. Qiu is with Central South University. She serves as the jointcorresponding author, email: Zhifeng.qiu@csu.edu.cn.

The research has been partially funded by KU Leuven internal funds (project C24/16/018). in LVDNs is managing a large group of users with ensuring (i) system constraints, (ii) user satisfaction, (iii) computational tractability [2].

Distributed control approaches are appealing to tackle these challenges. There are quite a few popular approaches that implement optimization in a distributed manner with communication [3], [4]. Model predictive control (MPC) [5]-[7] is a discrete time control approach, in which the control is not only determined by minimizing a cost function based on current state, but also finite steps of future time employing a predictive model. It can handle multi-variable control problems with tunable parameters, and explicitly consider the constraints. Consensus-based approaches [8], [9] are designed to solve distributed optimization problems by having different DERs converge to a common value. It can achieve global optimality via limited, time varying peer to peer communication, without needing a dedicated unit. Nevertheless, all of these approaches are iteration-based, the computational tractability cannot be guaranteed especially for users in LVDNs, as it is not realistic to expect users with high computational capability in LVDNs. Alternating direction method of multipliers (ADMM) [10], [11] decomposes the original optimization problem into a number of subproblems that are solved iteratively. It employs the augmented Lagrangian to improve convergence rate, whereas the convergence is sensitive to initialization when applied to non-convex optimal power flow (OPF) problems [12]. Moreover, modeling can be intractable in LVDNs, with multifarious users connected, continuously building new models does not effect a permanent cure. Therefore, model-free control [13] using a simplified representation of the system is preferable. In summary, a computationally tractable, modelfree approach that can conduct distributed optimization is needed to address the aforementioned challenges.

Although the communication hierarchy in distributed control approaches is decentralized, with the increase of users amount, some downsides appear: incomplete information acquisition, communication congestion, and significant increase in data refresh rate [4]. The proposed approach employs Mean Field Theory (MFT) [14] to tackle the issues when the user amount rises. With the growth of user amount, the influence and significance of individuals vanishes from the perspective of the system. MFT replaces the influence of all the other players on a given agent by their average influence, in order to efficiently approximate the collective behaviour of the group. MFT has found its application in engineering now and then, demand management employing MFT in smart grid is studied in [15], with a bang-bang switching control as its application. Readers are referred to [16] for specific 
engineering applications. Additionally, our previous work [17] discussed the demand management of shapeable loads in LVDN by MFT, whereas the uncertainties are not considered.

For every user $i$ in LVDN, the voltage control is regarded as a dynamic programming problem, and the corresponding optimization problem becomes a best response game. Namely, the nodal voltage $V_{t+1}^{i}$ at time $t+1$ depends on $V_{t}^{i}$ and on the control $u_{t}^{i}$, with the addition of the collective effect from all the other users. The optimal control $u_{t}^{i^{*}}$ is found by solving Hamilton-Jacobi-Bellman (HJB) function in this paper. In most of the applications, if not all, HJB function is combined with Fokker-Planck-Kolmogorov PDE [18], or Mc-Kean Vlasov SDE [19], in order to obtain the dynamical evolution of the group distribution. This leads to solving PDE sets iteratively. In this paper, the proposed approach employs a global broadcast to indicate the mean field information, together with some approximation, the iterations are streamlined. Moreover, the associated changes of reactive power are considered as uncertainty besides other uncertain factors, which will be discussed later on.

The main contribution of this paper is fourfold: firstly, a novel distributed approach to seek the approximation to optimal control in LVDN with considering uncertainty is proposed. A modified HJB equation is solved individually by each user to give the optimal control in a model-free manner without relying on iterations. Secondly, a broadcasterusers structure is proposed, to streamline the PDE sets that need to be solved by users. This makes the individual optimization problems computationally tractable, resulting in a balanced burden between communication and computation in practical applications. Thirdly, the ways to indicate different uncertainties in LVDN and their influences on the control are discussed from the mathematical perspective. Last but not least, a problem segmentation and adaptive feasible domain mechanism are proposed. It helps to implement the proposed control among users with different computational capabilities, and consequently makes it more practical. The paper is organized as follows: In section II the mathematical methodology is briefly elaborated, then the voltage control problem and the corresponding control scheme are presented in section III, section IV shows the results of case study and section $\mathrm{V}$ concludes the paper.

\section{MAthematicAl Methodology}

Consider an interacting system with $n$ users. The set of users is denoted by $N=\{1,2, \ldots, n\}$, and the time is denoted by $t \in\left[t_{0}, t_{0}+T\right]$, while $T>0$ is the length of a typical control period. The state of user $i \in N$ at $t$ is $x_{t}^{i}$, then the dynamic equation describing the evolution of user $i$ can be described as a SDE

$$
d x_{t}^{i}=\left[\alpha_{t} x_{t}^{i}+\beta_{t} u_{t}^{i}\right] d t+\beta_{t} W x_{t}^{i} d w_{t}^{i},
$$

where $u_{t}^{i}$ is the admissible control at $t$ for user $i, \alpha_{t}, \beta_{t} \in \mathbb{R}$ are decay/growth parameters, and $w_{t}^{i}$ is a Wiener process with a multiplier $\mathrm{W}$ that depends on state $x_{t}^{i}$.

\section{A. Mean field theory}

Suppose that for each user $i$, there is a cost function $J_{t}^{i}$ related to its own state $x_{t}^{i}$ and the corresponding control $u_{t}^{i}$. The cost of $u_{t}^{i}$ depends on all the users' states $\left\{x_{t}^{i}\right\}$, which is described by a linear mapping $r_{i}(x)$. Then $J_{t}^{i}$ is given by

$$
J_{t}^{i}\left(x_{t}^{i}, u_{t}^{i}\right)=\mathbb{E}\left(l\left(x_{t}^{i}\right)+\sum_{j=1}^{n} r^{j}\left(\delta_{x_{t}^{i}}\right) u_{t}^{i^{2}}\right),
$$

where $\delta$ is the Dirac measure and $l\left(x_{t}^{i}\right)$ is the cost of $x_{t}^{i}$, $\mathbb{E}$ is the expectation. One can conclude from (2) that global communication is needed to compute $J_{t}^{i}$. Users interact with each other through a global cost function that is given by

$$
J_{t}=\mathbb{E}\left(\sum_{i=1}^{n} J_{t}^{i}\left(x_{t}^{i}, u_{t}^{i}\right)\right)=\mathbb{E}\left(\sum_{i=1}^{n} l\left(x_{t}^{i}\right)+\sum_{i=1}^{n} \sum_{j=1}^{n} r^{j}\left(\delta_{x_{t}^{i}}\right) u_{t}^{i^{2}}\right) \text {. }
$$

In addition to communication issues when users are numerous, it can cause privacy issues since detailed information needs to be accessible to every user. Nevertheless, the influence and significance of individuals vanish when the amount of users rises. Thus, the influence of all the other users is replaced by the average according to MFT. Namely, (2) can be written as

$$
J_{t}^{i}\left(x_{t}^{i}, u_{t}^{i}, \widetilde{m}_{t}^{-i}\right)=\mathbb{E}\left(l\left(x_{t}^{i}\right)+\bar{r}^{-i}\left(\widetilde{m}_{t}^{i}\right) u_{t}^{i^{2}}+r^{i}\left(\delta_{x_{t}^{i}}\right) u_{t}^{i^{2}}\right),
$$

where $\widetilde{m}_{t}^{-i}$ is given by

$$
\widetilde{m}_{t}^{-i}=\frac{1}{n-1} \sum_{\substack{i^{\prime}=1, i^{\prime} \neq i}}^{n} \delta_{x_{i^{\prime}, t}}
$$

Then (4) works as if user $i$ only interacts with one user, who is the representative of the collective effects. Further, (4) can be approximated by merging $\bar{r}^{-i}$ and $r^{i}$ as $\bar{r}$, then

$$
J_{t}^{i}\left(x_{t}^{i}, u_{t}^{i}, \bar{m}_{t}\right)=\mathbb{E}\left(l\left(x_{t}^{i}\right)+\bar{r}\left(\bar{m}_{t}\right) u_{t}^{i^{2}}\right),
$$

where $\bar{m}_{t}$ is given by

$$
\bar{m}_{t}=\frac{1}{n} \delta_{x_{t}^{i}}+\frac{n-1}{n} \widetilde{m}_{t}^{-i}
$$

Consequently, (3) becomes

$$
J_{t}=\sum_{i=1}^{n} J_{t}^{i}\left(x_{t}^{i}, u_{t}^{i}, \bar{m}_{t}\right)=\sum_{i=1}^{n} \mathbb{E}\left(l\left(x_{t}^{i}\right)+\bar{r}\left(\bar{m}_{t}\right) u_{t}^{i^{2}}\right) .
$$

\section{B. Optimal response of user $i$ with uncertainty}

Consider a system described by (1) with an initial state $\left\{x_{0}^{i}\right\} \cdot\left\{x_{0}^{i}\right\}$ follows an initial distribution $m_{0}^{i}$ which converges almost surely to $m_{0}$ if $n \longrightarrow \infty$. According to Itô's formula, $m_{t}$ with initial condition $m_{0}$ is a weak solution of the FokkerPlanck-Kolmogorov forward equation, which is given by

$\partial_{t} m_{t}(x)+\partial_{x}\left[\left(\alpha_{t} x+\beta_{t} u^{*}(x)\right) m_{t}(x)\right]-\frac{\beta_{t}^{2} W^{2}}{2} \partial_{x x}^{2}\left[x^{2} m_{t}(x)\right]=0$,

for all $t \in(0, T]$. And $m_{0}$ is given.

User $i$ aims to figure out the admissible control $u_{t}^{i}$ that always respects the state constraints and minimizes the expected cost. Then during $t \in(0, T]$, the cost of user $i$ is given by

$$
J^{i}\left(x_{0}^{i}, x_{t}^{i}, u_{t}^{i}, m_{t}\right)=\mathbb{E}\left(\int_{0}^{T}\left[l\left(x_{t}^{i}\right)+\bar{r}\left(\bar{m}_{t}\right) u_{t}^{i^{2}}\right] d t .\right.
$$


The infimum of (10) is defined as a value function $v_{t}(x)$, as

$$
v_{t}(x)=\inf _{u} \int_{0}^{T}\left[l\left(x_{t}^{i}\right)+\bar{r}\left(\bar{m}_{t}\right) u_{t}^{i^{2}}\right] d t .
$$

Assume that $\beta_{t} \neq 0$, the Hamiltonian is given by

$$
H_{t}\left(x_{t}^{i}, m_{t}\right)=\inf _{u}\left\{J_{t}^{i}\left(x_{t}^{i}, u_{t}^{i}, m_{t}\right)+\partial_{x} v_{t}(x)\left(\alpha_{t} x_{t}^{i}+\beta_{t} u_{t}^{i}\right)\right\},
$$

where the $v_{t}(x)$ satisfies the HJB-Flemming equation

$$
\partial_{t} v_{t}(x)+H_{t}\left(x_{t}^{i}, m_{t}\right)+\frac{\beta_{t}^{2} W^{2} x^{2}}{2} \partial_{x x}^{2} v_{t}(x)=0 .
$$

Assuming strict convexity of $J_{t}^{i}$ in $u$, then from (12), it can be derived that

$$
\partial_{u} J_{t}^{i}\left(x_{t}^{i}, u_{t}^{i^{*}}, m_{t}\right)+\partial_{x} v_{t}(x) \beta_{t}=0 .
$$

Plug (6) into (14), then the optimal control is given by

$$
u_{t}^{i^{*}}=-\frac{\partial_{x} v_{t}(x) \beta_{t}}{2 \bar{r}\left(\bar{m}_{t}\right)} .
$$

The properties of the optimal response of user $i$ are given by

$$
\left.\begin{array}{r}
\partial_{t} v_{t}(x)+l\left(x_{t}^{i}\right)+\bar{r}\left(\bar{m}_{t}\right) u_{t}^{i^{* 2}}+\partial_{x} v_{t}(x)\left(\alpha_{t} x_{t}^{i}\right. \\
\left.+\beta_{t} u_{t}^{i^{*}}\right)+\frac{\beta_{t}^{2} W^{2} x^{2}}{2} \partial_{x x}^{2} v_{t}(x)=0 \\
\partial_{t} m_{t}(x)+\partial_{x}\left[\left(\alpha_{t} x+\beta_{t} u^{*}(x)\right) m_{t}(x)\right] \\
-\frac{\beta_{t}^{2} W^{2}}{2} \partial_{x x}^{2}\left[x^{2} m_{t}(x)\right]=0 \\
u_{t}^{i^{*}}=-\frac{\partial_{x} v_{t}(x) \beta_{t}}{2 \bar{r}\left(\bar{m}_{t}\right)} \\
m_{0}=m_{t_{0}}^{i}
\end{array}\right\},
$$

which is a PDE set that consists of a HJB-Flemming PDE and a Fokker-Plank PDE. Fokker-Planck equation describes the time evolution of the probability density function of the velocity of a particle under the influence of drag forces and random forces, and it can be generalized to other observables as well [20]. Conventionally, it is used to estimate the system evolution after $u_{t}^{i *}$ is implemented. The two PDEs in (16) can be solved iteratively to seek the optimal trajectory and the corresponding $u_{t}^{i *}$, which is computational intensive. In order to reduce the computational burden, the Fokker-Planck PDE in (16) is replaced by broadcast signal in the proposed approach, which will be elaborated in the control scheme section. For more preliminaries and detailed derivatives of this section, readers are referred to book [21].

Remarks 1: There are two ways to the entry of uncertainties. The first one is equation (1), in which there is a Wiener process defined on $(\Sigma, \mathcal{F}, \mathbb{P})$, where $\mathcal{F}$ is the nature filtration generated by $W_{t}^{i}$. From the perspective of computation, this results in the quadratic partial differential part of (9) and (13) as a result of Itô's calculus. And eventually reaches (16).

The second one is a disturbance entering together with $u_{t}^{i}$. Assume the disturbance is $\zeta_{t}^{i}$, then (1) becomes

$$
d x_{t}^{i}=\left[\alpha_{t} x_{t}^{i}+\beta_{t} u_{t}^{i}+\sigma_{t} \zeta_{t}^{i}\right] d t .
$$

Consequently, (6) changes to

$$
J_{t}^{i}\left(x_{t}^{i}, u_{t}^{i}, \bar{m}_{t}\right)=\mathbb{E}\left(l\left(x_{t}^{i}\right)+\bar{r}\left(\bar{m}_{t}\right) u_{t}^{i^{2}}-\gamma^{2} \zeta_{t}^{i^{2}}\right),
$$

where $\gamma$ is a nonzero quadratic penalty on the disturbance coefficient $\sigma$. Then (12) becomes

$$
\begin{aligned}
\tilde{H}_{t}\left(x_{t}^{i}, m_{t}\right)= & \inf _{u} \sup _{\zeta}\left\{J_{t}^{i}\left(x_{t}^{i}, u_{t}^{i}, m_{t}\right)-\gamma^{2} \zeta_{t}^{i^{2}}+\right. \\
& \left.\partial_{x} v_{t}(x)\left(\alpha_{t} x_{t}^{i}+\beta_{t} u_{t}^{i}+\sigma_{t} \zeta_{t}^{i}\right)\right\}
\end{aligned}
$$

in which the function $-\gamma^{2} \zeta_{t}^{i^{2}}+\partial_{x} v_{t}(x) \sigma_{t} \zeta_{t}^{i}$ is concave. Therefore its maximizer is given by

$$
\zeta_{t}^{i^{*}}=\frac{\sigma_{t}}{2 \gamma^{2}} \partial_{x} v_{t}(x),
$$

which indicates the worst case of disturbance. Then, (17), (18) and (19) become deterministic by plugging (20), replacing $\zeta_{t}^{i}$ by its maximum. For more details and derivatives, readers are referred to robust Nash equilibrium in [22].

The uncertainty introduced by Wiener process can be used to represent stochastic or uncontrollable factors in LVDN, for instance, the uncertainty of the renewable generations. While the entry of uncertainty via control $u_{t}^{i}$ stands for disturbances in communication or operation. This kind of uncertainty can be replaced by its worst case, then the corresponding functions can be solved in their deterministic form.

\section{CONTROL SCHEME}

\section{A. Problem statement}

Consider a LVDN with $n>100$ multifarious users including normal households, households with PV, small residential wind turbines, and other distributed generators. The nodal voltages need to be actively regulated in the context of ADN. Each user has a certain degree of flexibility, which varies with time due to different user habits. Due to privacy concerns, users are not willing to share their electricity consumption data. Although it might be a bit severe, this is a representative case for LVDN in the future. [23]. From the perspective of regulation, all the nodal voltages have to be kept within the system constraints. Since voltage regulation consumes flexibility, which affects users' comfort, the control needs to be optimized in order to implement voltage regulation with respecting every user's scheduled consumption as much as possible. Namely, the control need to minimize

$$
C_{t}=\sum_{i=1}^{n}\left(x_{t}^{i^{\prime}}-x_{t}^{i}\right)^{2},
$$

where $x_{t}^{i}$ is the actual consumption of user $i$ while $x_{t}^{i}{ }^{\prime}$ is the corresponding scheduled consumption profile.

\section{B. Public information setup}

Public information is crucial in distributed control. Normally, users need public information to make sure that they are on the same page in one control period. For instance, in classical droop control, frequency is employed as public information among users. In this paper, a broadcaster is used to offer public information $\bar{m}_{t}$. More specifically, the broadcaster collects nodal voltages from evenly allocated key monitoring 
nodes in LVDN, then computes the average voltage $\bar{V}_{t}$ and generates the public signal $\xi_{t}$ by

$$
\xi_{t}=\left(1-\sin \left(\frac{e^{-\left(\frac{\mu}{10}\right)^{2}}}{2} \pi\right)\right) \cdot \operatorname{sgn}(\mu), \text { where } \mu=\bar{V}_{t}-V^{r},
$$

where $V^{r}$ is the nominal voltage. Note that the key nodes are different from user nodes. As only the average voltage is needed, it is not necessary to measure all the nodal voltages. $\xi_{t}$ is used to indicate the general situation of the LVDN, and there is no high demand on its accuracy. It is generated and broadcast to all the users periodically with an interval of $T$.

Remarks 2: The broadcast of public information is the only communication needed in the proposed approach, which is suitable for large scale deployment. There are three aspects to it. i) Bandwidth. The only message need to be delivered in the proposed approach is $\xi_{t}$, which is a plain single-digit value. In centralized optimization [24], more information such as working status, voltage measurements, scheduled consumption profiles need to be delivered between users and the controller, which demands higher bandwidth. Moreover, distributed approaches do not necessarily mean low bandwidth occupation. For instance, peer to peer gossip based ADMM [25] requires multipliers exchange among users, users in push-pull distributed optimization [26] keep exchanging their matrix. ii) Communication architecture. Broadcast is an unidirectional, single blind communication. Compared to point to point communication which is widely used in most of the existing approaches, broadcast is more flexible as users can join/exit freely. The communication network can easily be implemented by wireless communication. iii) Communication burden while number of users increases. Broadcast is insensitive to the number of users, as it is an indiscriminate communication. As contrasted, centralized communication is sensitive to the number of users, assume user number is $n$ and message length per communication is $e$, then the communication burden of centralized communication is $2 \mathrm{en}$. And the communication burden of the peer to peer communication in [25] depends on the topology and size of the network. Additionally, it is reasonable to question the dependability of the broadcast architecture. Therefore, communication uncertainty is also considered, the robustness test on communication latency and packet loss are provide as special cases in section IV later on.

\section{Approximation to optimal response of user $i$}

1) Evolution function: Let $\alpha_{t}=0$ and $\beta_{t}=1$ in (1). Then (1) becomes

$$
d \hat{x_{t}^{i}}=u_{t}^{i} d t+x_{t}^{i} W d w_{t}^{i}, \text { with } d x_{t}^{i}=u_{t}^{i} d t,
$$

where $d \hat{x_{t}^{i}}$ is composed by control $u_{t}^{i}$ and the introduced uncertainty. $\hat{x}_{t}^{i}$ is defined as "equivalent active power", which is different from practical active power $x_{t}^{i}$. This definition comes by assuming the voltage in LVDN is only related to active power. This assumption is established on two facts. Firstly, in common LVDN, R/X ratio is so high that voltage is more sensitive to the changes on active power rather than reactive power [27]. Secondly, most of the users in LVDN lack a feasible way to control their reactive power consumption.
For instance, appliances in households like washing machine or EV charging station might reshape their consumption due to active power regulation, but reactive power regulation can barely be possible. Some power electronic devices in LVDN are able to control their reactive power, yet the standards need to be established [28]. Nevertheless, when active power consumption changes, the reactive power consumption changes accordingly. With the concept of equivalent active power, voltage control in LVDN is converted as active power control with uncertainty in (23). Specifically, $u_{t}^{i}$ is the control that aims to $x_{t}^{i}, w_{t}^{i}$ is the associated changes on reactive power. Then $\hat{x}_{t}^{i}$ is the equivalent active power as if the voltage only relates to active power.

2) Cost function: With specific $l$ and $\bar{r}$, (6) becomes

$$
J_{t}^{i}\left(x_{t}^{i}, u_{t}^{i}, \xi_{t}, \gamma_{t}^{i}\right)=\left(x_{t}^{i}-x_{t}^{i^{\prime}}\right)^{2}+e^{\varphi_{t}^{i}} u_{t}^{i^{2}} .
$$

$x_{t}^{i^{\prime}}$ is the planned active power consumption according to the load profile of user $i$, and $\varphi_{t}^{i}$ is given by

$$
\varphi_{t}^{i}=\left\{\begin{array}{ll}
2-\left|\Theta_{t}\right| & ,\left(x_{t}^{i^{\prime}}-x_{t}^{i}\right) \Theta_{t} \geq 0 \\
1+5 \cdot\left|\Theta_{t}\right| & ,\left(x_{t}^{i^{\prime}}-x_{t}^{i}\right) \Theta_{t}<0
\end{array} .\right.
$$

And $\Theta_{t}$ is a coefficient that works in seesaw way to take balance between global and local information, given by

$$
\Theta_{t}=\left(1-\left|\gamma_{t}^{i}\right|\right) \xi_{t}+\left|\gamma_{t}^{i}\right| \cdot \gamma_{t}^{i}
$$

where it is similar to $\xi_{t}, \gamma_{t}^{i}$ is given by

$$
\gamma_{t}^{i}=\left(1-\sin \left(\frac{e^{-\left(\frac{\mu}{10}\right)^{2}}}{2} \pi\right)\right) \cdot \operatorname{sgn}(\mu), \quad \mu=V_{t}^{i}-V^{r} .
$$

$V_{t}^{i}$ is the nodal voltage of user $i$. Note the values 2 and 5 in (25) are empirical values and tunable, as long as it can make sure neither $u_{t}^{i^{2}}$ nor $\left(x_{t}^{i}-x_{t}^{i^{\prime}}\right)^{2}$ is dominating.

Eq. (24) consists of two parts: $\left(x_{t}^{i}-x_{t}^{i^{\prime}}\right)^{2}$ indicates the gap between the current active power consumption and scheduled active power consumption of user $i$, stimulated by the cost, user $i$ always wants to change $x_{t}^{i}$ towards $x_{t}^{i^{\prime}}$, and this can be done by $u_{t}^{i}$ in (23). This is in accordance with the cost function (21) that the given LVDN wants to minimize. However, from the perspective of dynamic programming in the proposed approach, in order to take the voltage constraints into account, $u_{t}^{i}$ does rise another part of the cost. According to (25), $u_{t}^{i}$ will be expensive if user $i$ changes $x_{t}^{i}$ towards the direction that will deteriorate the voltage situation, and the opposite holds as well. This mechanism encourages user $i$ to change $x_{t}^{i}$ towards the direction that fixes the voltage deviation, and sets barriers if the change enlarges the deviation. Large $\varphi_{t}^{i}$ leads to small $u_{t}^{i}$, which makes $d x_{t}^{i}$ so small that $x_{t}^{i}$ may not be able to reach $x_{t}^{i^{\prime}}$ within $\left(t_{0}, t_{0}+T\right]$. Moreover, large $\varphi_{t}^{i}$ drives the optimal point to deviate from $x_{t}^{i^{\prime}}$ as well. This mechanism generates the operation bounds for user $i$ respect to voltage limits without relying on specific models. 
3) HJB PDE of user $i$ : In order to simplify computation, $\xi_{t}$ is used to replace the solution of the second PDE in (16). The PDE that user $i$ needs to solve independently becomes

$$
\left.\begin{array}{r}
\partial_{t} v_{t}(x)+\left(x_{t}^{i}-x_{t}^{i^{\prime}}\right)^{2}+e^{\varphi_{t}^{i}} u_{t}^{i^{* 2}}+\partial_{x} v_{t}(x)\left(\beta_{t} u_{t}^{i^{*}}\right) \\
+\frac{\beta_{t}^{2} W^{2} x^{2}}{2} \partial_{x x}^{2} v_{t}(x)=0 \\
u_{t}^{i^{*}}=-\frac{\partial_{x} v_{t}(x)}{2 e^{\varphi_{t}^{i}}} \\
x_{0}=x_{t_{0}}^{i} \\
x_{t}^{i} \in\left[x_{i}^{\text {min }}, x_{i}^{\text {max }}\right]
\end{array}\right\} .
$$

An approximation is made in (28), where user $i$ assumes $\xi_{t}$ remains unchanged during $\left(t_{0}, t_{0}+T\right]$. Practically, as long as user $i$ makes change, $\xi_{t}$ changes. In order to reduce communication burden, $\xi_{i}$ is only updated every $T$ time. Nevertheless, this approximation will not result in significant error, as according to (26), $\varphi_{t}^{i}$ is determined by $\xi_{t}$ and $\gamma_{t}^{i}$. Although $\xi_{t}$ is not updated timely, user $i$ is able to update $\gamma_{t}^{i}$ easily. This can be done by an asynchronous control mechanism. In a given LVDN, it is likely that not all the users have same computational capability. For instance, user $i$ may have an older controller than user $j$, which takes user $i$ more time to solve its (28). Asynchronism among users could be problematic in some control schemes, whereas the proposed scheme takes advantage of it to a better approximation. During $\left(t_{0}, t_{0}+T\right]$, user $i$ needs to solve (28) and implement $u_{t}^{i *}$. Upon its computation capability, (28) can be solved multiple times with the updated $\gamma_{t}^{i}$. Although the total adjustment always is $\int_{0}^{T} u_{t}^{i^{*}} d t, u_{t}^{i *}$ can be updated multiple times. The more PDEs user $i$ solves, the closer its control trajectory to optimum.

4) Problem segmentation: From the perspective of implementation, solving HJB PDE is not an easy task. First and foremost, obtaining exact numerical solution of HJB function is still an academic issue, most of the solving mechanisms existing seek the approximation to viscosity solution instead [29]. Rutquist [30] proposed an approach to seek the approximate numerical solution of HJB equation with state constraints, which demands much less collocation points to give a good approximation, reduces the computing power requirements.

Although it is not common, for (28), the solving process can demand finer mesh or large number of collocation points if it has a large feasible domain together with a large initial condition $\left(x_{t_{0}}^{i}-x_{t}^{i^{\prime}}\right)$. Large amount of collocation points inevitably leads to knotty computational complexity even if it gets relieved by [30]. If it takes significantly long time to solve one PDE, a user may have no sufficient time to implement the control or come up with considerable error. To tackle this, the optimization problem is segmented into several small continuous problems if the original one is too large. A numerical test is stated by the Table I in [30], which suggests a proper amount below 64 for collocation points. Therefore, an empirical limit of 5 is given to $\left(x_{t_{0}}^{i}-x_{t}^{i^{\prime}}\right)$. Note this limit is proportional, its absolute value depends on its unit. As the instance in Fig. 1, if in $\left(t_{0}, t_{0}+T\right], x_{t_{0}}^{i}$ is 2 and the corresponding $x_{t}^{i^{\prime}}$ is 9 , (27) will be divided into two smaller problems with $x_{t_{0}}^{i}=2, x_{t}^{i^{\prime}}=7$ and $x_{t_{0}}^{i}=7, x_{t}^{i^{\prime}}=9$

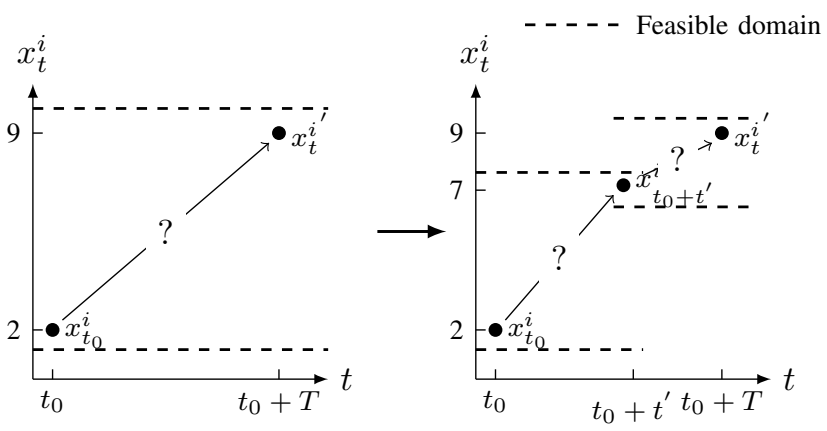

Fig. 1. Problem segmentation

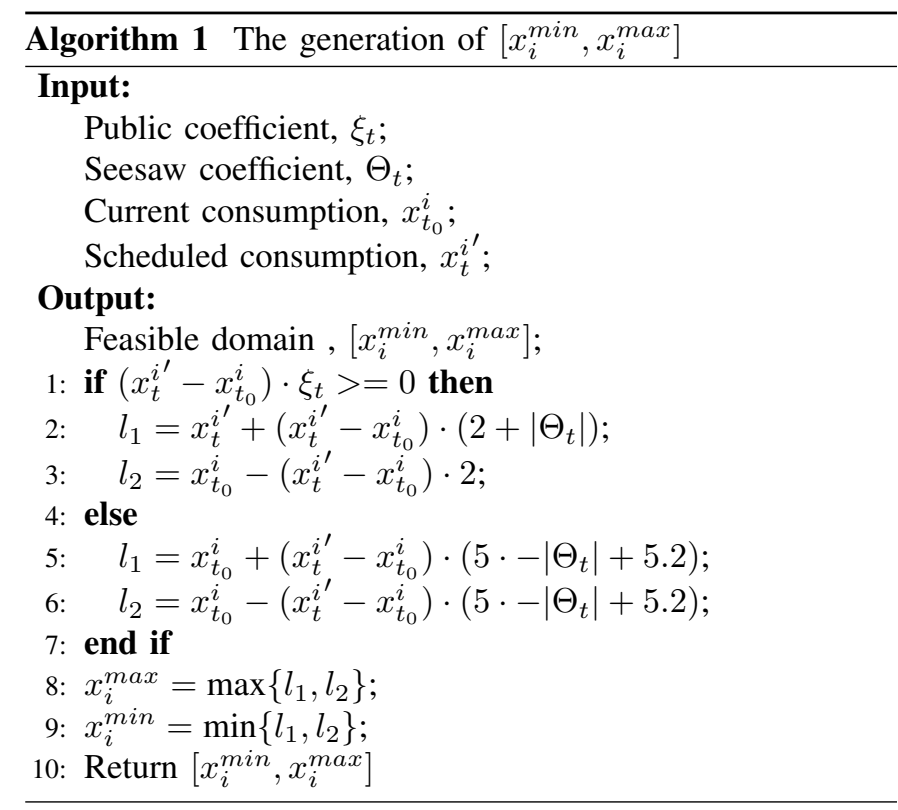

respectively. Then they will be solved and implemented in sequence with updated $\gamma_{t}^{i}$.

The segmented problems do not share the same feasible domain . The corresponding $\left[x_{i}^{\min }, x_{i}^{\max }\right]$ are given by Algorithm 1, where the feasible domain depends on $\xi_{t}$ and $\Theta_{t}$. This leads to a flexible feasible domain requiring less collocation points so that the PDE of segmented problems can be solved easier and faster. It can be concluded from Algorithm 1 that when the scheduled change is contrary to what LVDN needs, the feasible domain shrinks upon $\xi_{t}$ and $\Theta_{t}$. From the perspective of computation, the control trajectory will gradually converge to the median if the feasible domain is narrow. Therefore, with $\Theta_{t}$ approaching its bounds, $x_{t}^{i}$ is "locked" by a virtual bound. This mechanism is useful as due to the lack of explicit model that indicates the relationship between voltage and power flow, nodal voltage might exceed its boundaries if there is a dramatic change on the corresponding power profile. This is not an issue to the proposed approach, since the feasible domain of (28) shrinks according to $\Theta_{t}$, which exerts virtual boundary to stop user $i$ from exceeding the physical voltage bounds. This segmentation mechanism helps user $i$ solve PDEs faster, which yields a better approximation to the optimal control trajectory as aforementioned. 


\section{CASE STUdy}

\section{A. Grid Topology and profiles}

The schematic diagram is illustrated in Fig. 2. It is a three phase 230/400V reference grid, based on the topology of a real semiurban feeder in Flanders, Belgium. All of the main feeder cables are of type EAXVB $1 \mathrm{kV} 4 \cdot 150 \mathrm{~mm}^{2}$. A 250 $\mathrm{kVA} 10 / 0.4 \mathrm{kV}$ transformer is assumed with an impedance of $0.013+0.038 \mathrm{j}$ pu. From feeders to each individual user, a cable EXVB $1 \mathrm{kV} 4 \cdot 16 \mathrm{~mm}^{2}$ is used with a length of $15 \mathrm{~m}$. The nominal line-to-neutral voltage $V^{r}$ of all the users is $230 \mathrm{~V}$. The cable parameters are given in Table I, while the technical operating ranges of user consumption $x_{t}^{i}$ are given in Table II. To make the network multifarious and ill-designed, new users such as residential wind turbines and small PV farms are added, increasing the number of nodes from 62 to 103 . A model of domestic electricity use is used to generate high resolution household consumption profile, profiles of $\mathrm{PV}$ are taken from a $368 k W p$ PV system on the rooftop of a energy research institute in Genk, Belgium. All the engineering details about the testing network can be found in section 4.1 and 4.2 in [31].

TABLE I

CABLE PARAMETERS

\begin{tabular}{|c|c|}
\hline Cable Type & Impedance at $45^{\circ} \mathrm{C}$ \\
\hline EAXVB $1 \mathrm{kV} 4 \cdot 150 \mathrm{~mm}^{2}$ & $0.227+0.078 \mathrm{j} \Omega / \mathrm{km}$ \\
EXVB $1 \mathrm{kV} 4 \cdot 16 \mathrm{~mm}^{2}$ & $1.265+0.083 \mathrm{j} \Omega / \mathrm{km}$ \\
\hline
\end{tabular}

TABLE II

OPERATING RANGES OF USERS IN TEST NETWORK

\begin{tabular}{|c|c|}
\hline User Type & Operating Range $(\mathrm{kW})$ \\
\hline Houses without PV & $0 \sim 13$ \\
Houses with PV & $-4 \sim 13$ \\
Small PV farms & $-20 \sim 0$ \\
Residential wind turbines & $-11.5 \sim 0$ \\
High-consuming unit & $0 \sim 17$ \\
Residential generators & $-7 \sim 0$ \\
\hline
\end{tabular}

\section{B. Simulation setup}

The simulation of the proposed approach is implemented in Matlab 2019a on an Intel I7-6600U CPU @ 2.6 GHz, with toolboxes SNOPT and PROPT from Tomlab. The power flow simulation is based on the backward-forward sweep method [32]. Meanwhile, a centralized control [24] that derives the optimal adjustment for each participating user in LVDNs is implemented for benchmark purpose. The benchmark is coded in Julia, with the distribution network simulation package developed by the Los Alamos National Lab [33]. The power flow simulation programs used in Matlab and Julia are cross validated by OpenDSS to guarantee the consistency. In the benchmark algorithm, the controller solves an optimization problem using the full non-linear network model (Alternating Current Optimal Power Flow, ACOPF) to minimize (21). It employs bidirectional communication, each participating user sends a range to the central controller, within which it can adjust its power consumption or generation. Besides, the assumptions of ideal operating condition including perfect topology knowledge, real-time measurement, instant computation, communication and implementation are given, in order to obtain the theoretical best result to be compared with. It has same objectives with the proposed approach, except the cost of control, as cost of control is a virtual concept in the proposed approach to compute control bounds without explicit models.

\section{Results and remarks}

The average voltages that yield $\xi_{t}$ are illustrated in Fig. 3, in order to deliver an overall view between the original profile and the control schemes. In this case the reactive power is assumed to be compensated by external devices completely, and $W=0.1$ in the proposed approach. All the users are set to be able to solve three PDEs per control period, and $T=60 s$. The average PDE solving time of each user is 0.654 second. This scenario is to show the gap between the proposed approach and the theoretical optimum. From Fig. 3, it is reasonable to conclude that the proposed approach has managed the given LVDN decently, delivering very similar result to the benchmark approach. A notable gap appeared when the original profile leaded to a severe overvoltage: the benchmark approach can precisely confine the voltage just within the boundary, while the proposed approach is a bit more conservative, which means it consumes relatively more flexibility. A closer view is given by nodal voltages in Fig. 4, which clearly shows that the proposed approach was relatively more conservative when voltages approach technical bounds. This phenomenon comes from the different regulation mechanisms between two approaches. Since the ACOPF has an explicit model to indicate the relationship between power flow and voltage distribution, it can figure out more precise results, while the proposed approach is model-free, in which the regulation is carried out with the "virtual bounds" elaborated in section 3.C.4. Additionally, it is reasonable to doubt whether the proposed approach actually converged to a similar local optimum instead of the global one. Some hints can be obtained from Fig. 5, which presents the total power generation and consumption with original profile, proposed approach and ACOPF respectively. One can see that the proposed approach indeed delivered a good approximation to the theoretical optimum.

In order to compare with the benchmark approach numerically, we propose dissatisfaction rate as key performance indicator, which is given by

$$
D_{t}=\sum_{i=1}^{n}\left|x_{t}^{i}-x_{t}^{i^{\prime}}\right|
$$

And the result is illustrated in Fig. 6, from which one can observe that the notable differences on dissatisfaction rate take place during the period when profile is relatively mild. This is because the virtual bounds mechanism is a continuous process which starts as long as $V_{t}^{i}$ is far from $V^{r}$, the regulation takes place earlier in the proposed approach as aforementioned. The 


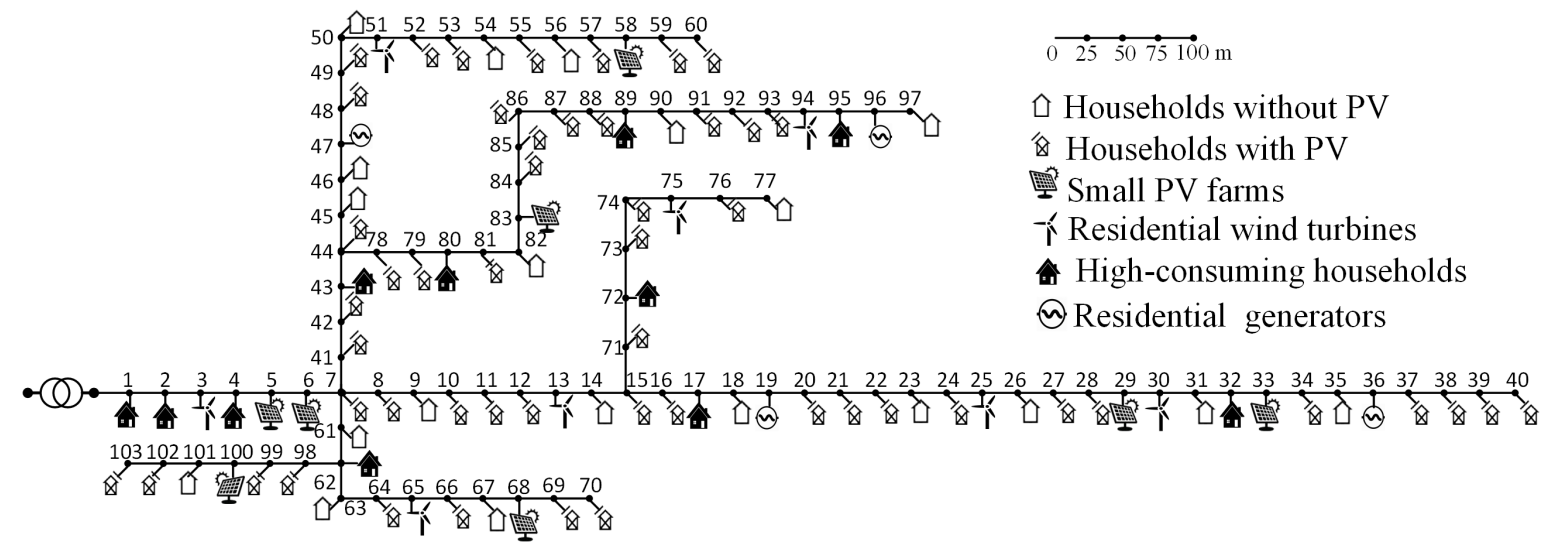

Fig. 2. Topology of the test network. Cable lengths are drawn to scale.

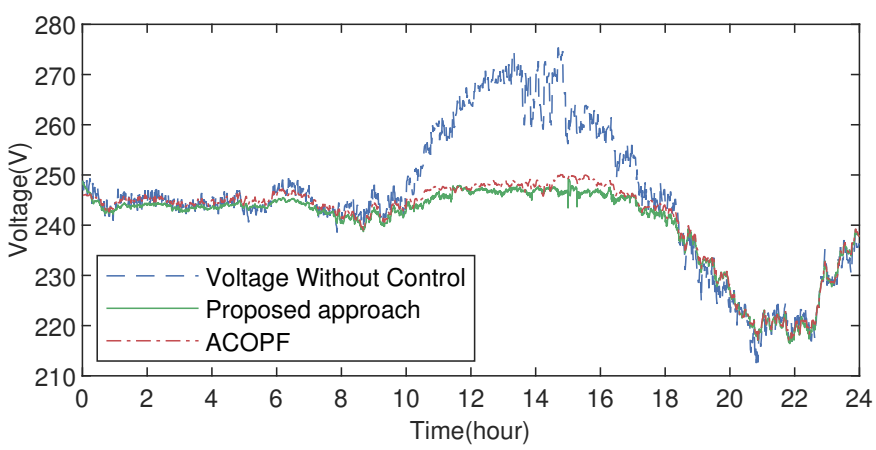

Fig. 3. General average of nodal voltages

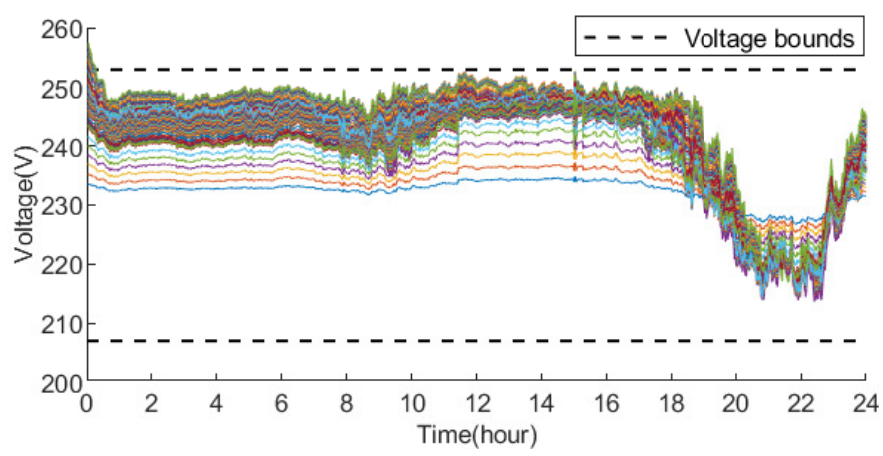

(a) Nodal voltages of the proposed approach

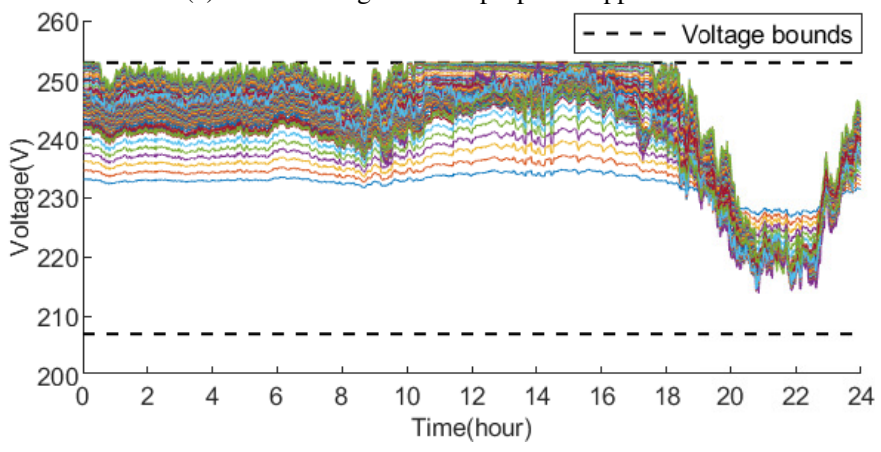

(b) Nodal voltages of ACOPF

Fig. 4. Evolvement of nodal voltages

average $D_{t}$ of the ACOPF is 6.8 and 9.1 for the proposed approach, which is an acceptable gap. It is fair to claim that

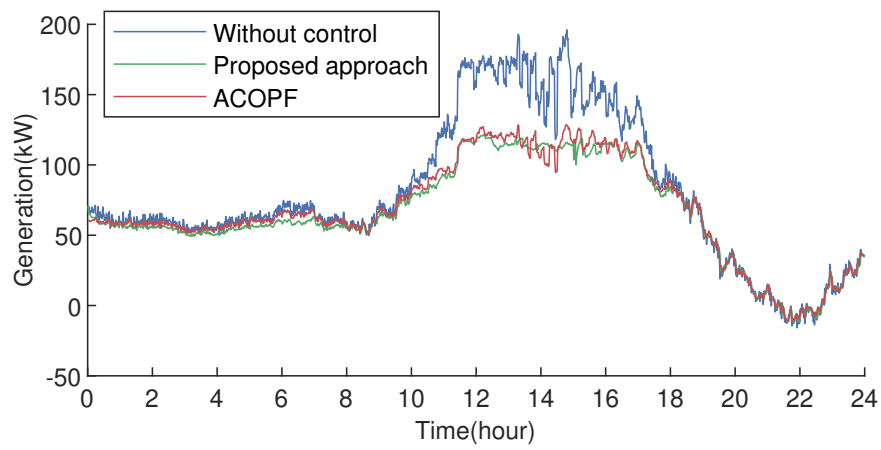

(a) Total power generation

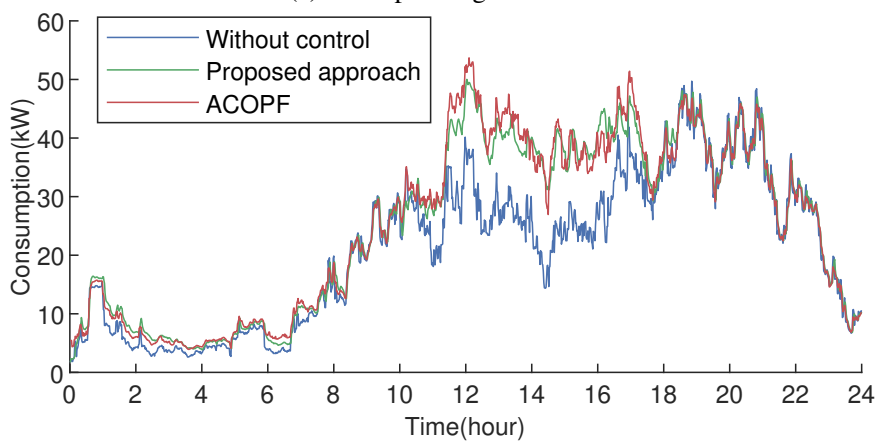

(b) Total power consumption

Fig. 5. Comparison of total power generation and consumption

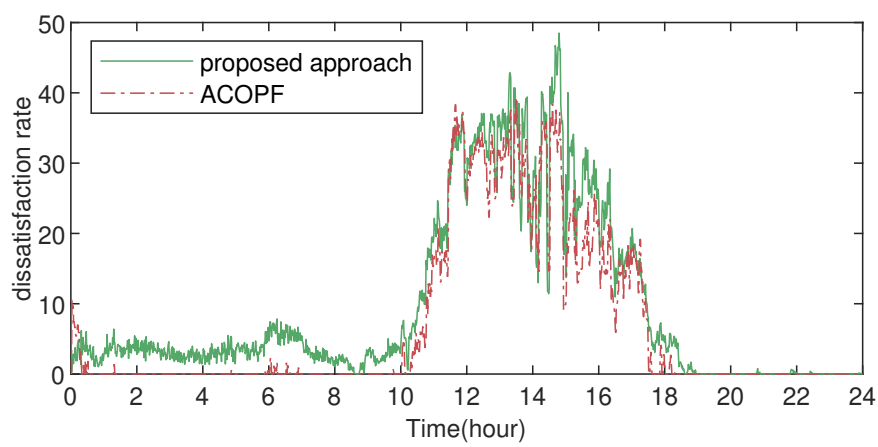

Fig. 6. Dissatisfaction rate without uncertainty

the proposed approach yields a good approximation to the optimum in model-free and distributed manner. 


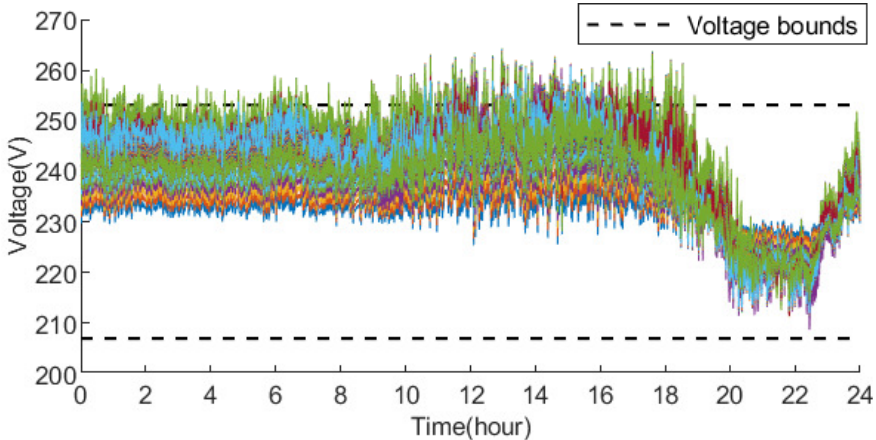

(a) The original ACOPF

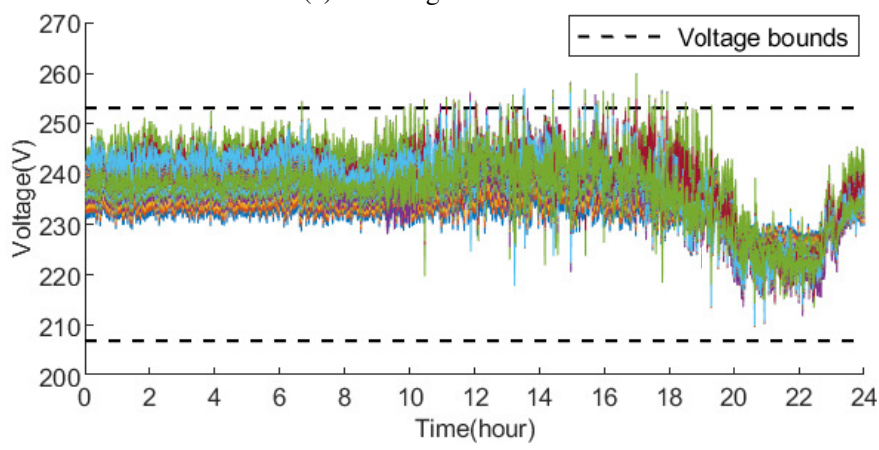

(b) Proposed approach with $\mathrm{W}=4$

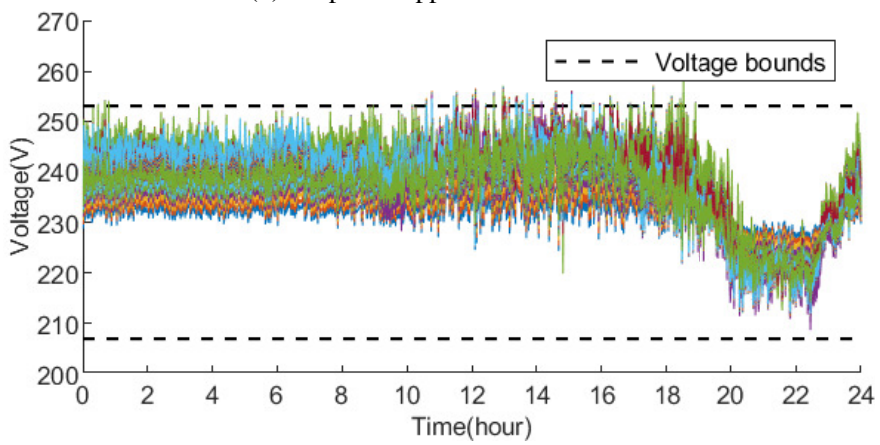

(c) ACOPF with modified bounds of $\pm 7 \%$

Fig. 7. Evolvement of nodal voltages with reactive power as uncertainty

If the reactive power is not compensated by external devices, the scenario comes to voltage control under uncertainty. In this case it is assumed that the power factor changes within $\pm[0.75,1]$ randomly as long as there is a control exerted on users. Although this is a harsh and unrealistic scenario, it is a good testing case for the performances under uncertainty. For simplicity, the average voltage figure is omitted, while the nodal voltages are shown in Fig. 7. It can be observed from Fig. 7(a) that the benchmark ACOPF cannot manage the nodal voltages properly anymore under such a harsh uncertainty, as there is no explicit model to indicate how the reactive power changes and its influence on the corresponding nodal voltages. Contrast to this, as shown in Fig. 7(b), the proposed approach is still able to manage the nodal voltage most of the time. There are three reasons to it. Firstly, with a larger $W$, the proposed approach becomes more conservative, which leads to more margin in control. Secondly, during $\left(t_{0}, t_{0}+T\right)$, user $i$ reviews the corresponding nodal voltage $V_{t}^{i}$ as elaborated in section 3.C.4. This allows users review the actual voltage changes and correct the control correspondingly. Last but not

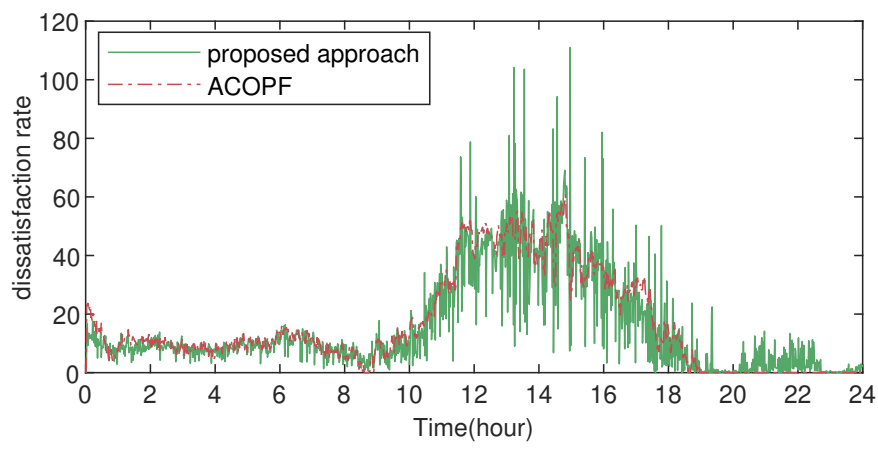

Fig. 8. Dissatisfaction rate with uncertainty

least, as the control is decentralized, each user only has to deal with its own uncertainty via its own HJB PDE, which makes the control personalized. Nevertheless, note the ACOPF can employ more margin to deal with the uncertainty as well. For doing this, its objective function need to be modified. Instead of allowing nodal voltage goes up to $\pm 10 \%$ deviation from $V^{r}$, a new deviation tolerance of $\pm 7 \%$ of $V^{r}$ is placed and the result is illustrated in Fig. 7(c). After enlarging the margin, the modified ACOPF yields a overvoltage rate $0.69 \%$ verses the proposed approach's $0.67 \%$, which is acceptable. Moreover, illustrated in Fig. 8, the modified ACOPF yields an average $D_{t}$ of 16.17 , which is higher than the proposed approach's 15.36. The reason can be observed from Fig. 8. The $D_{t}$ of the proposed approach fluctuates along the changes caused by uncertainty, implying that besides keeping a conservative margin, the proposed approach responds to the uncertainty by updating its control. Yet the benchmark ACOPF only deals with the uncertainty by employing more margin.

\section{Special scenarios}

In order to assess the effectiveness of the proposed approach in realistic operation scenarios, some common issues in daily operations are studied as special scenarios. The performance of the proposed approach is illustrated with necessary analysis and benchmarks.

1) Critical system status: With the high penetration of renewable energy in LVDNs, the most common critical system status are severe overvoltage and overloaded lines [34]. Since usually the capacity of cables is considered in the network planning phase and managed by protection algorithms [35], severe overvoltage is chosen as the special scenario for testing in this paper. Fig. 9 illustrates the scenario that the proposed approach starts from the midday, where the test network is suffering from severe overvoltage. Fig. 9(a) shows the average of nodal voltages, in order to deliver a general idea about the operations of the network without control, the network with full management of the proposed approach and the network with proposed approach started from the critical system status. The test network is simulated with $\mathrm{PF} \in[0.9,1]$. In order to give clear comparison, $\mathrm{PF}$ is assumed to be same for user $i$ at the same time $t$ among the three operations. Fig. 9(b) illustrates all the nodal voltages of the given LVDN without control, from which one can see the given LVDN 


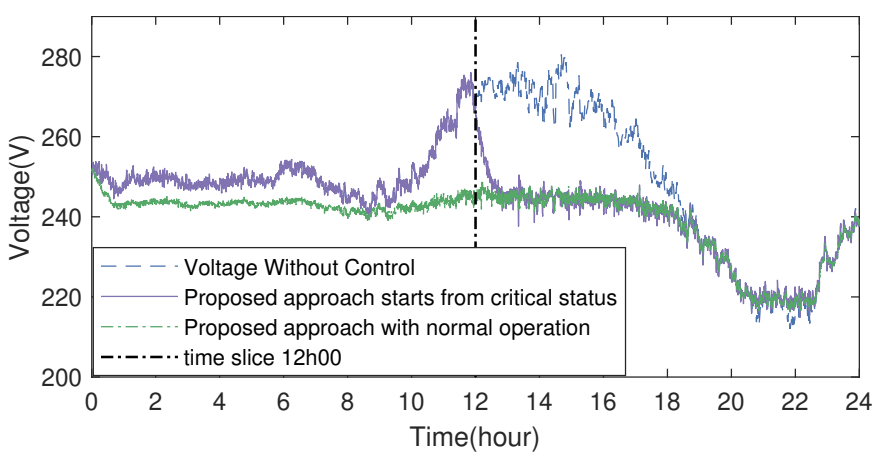

(a) The general average nodal voltage

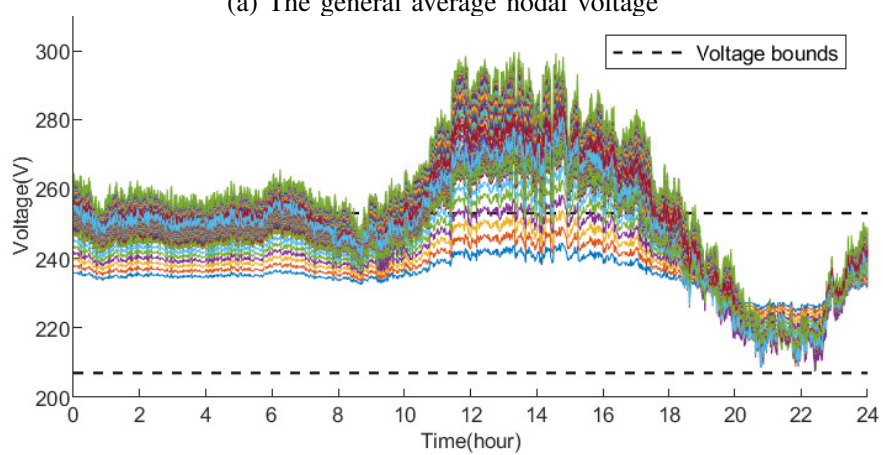

(b) Nodal voltages without control

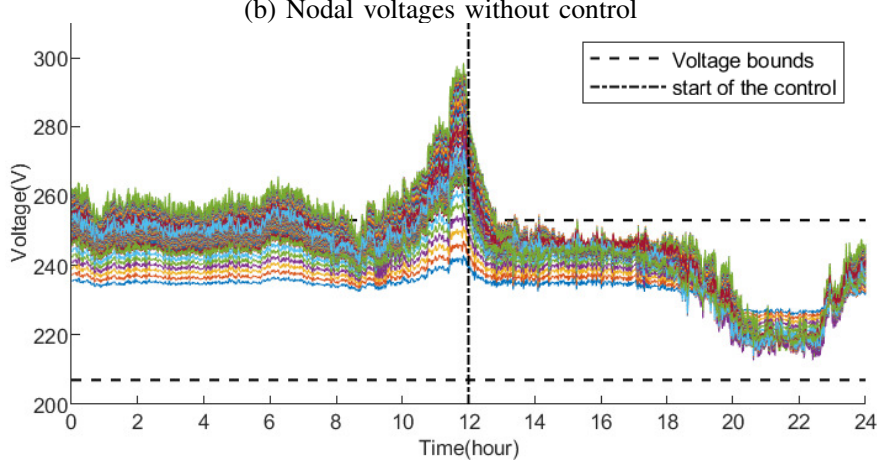

(c) Nodal voltages starts from the critical system status

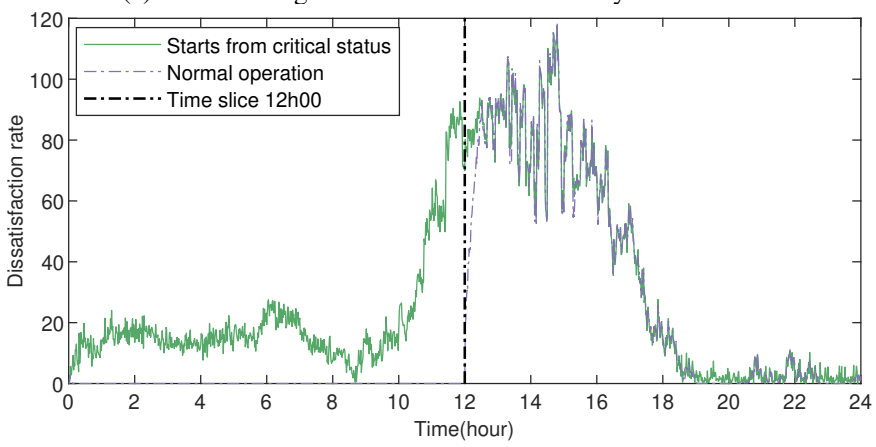

(d) Comparison on the dissatisfaction rates

Fig. 9. Special scenario of Critical system status

suffers from severe overvoltage issue if proper control is not implemented. Fig. 9(c) shows the nodal voltages that the proposed approach is implemented at the time $12 \mathrm{~h} 00$, where the give LVDN is under critical overvoltage status. Together with Fig. 9(d), one can conclude that the proposed approach is able to regulate the system from critical overvoltage status to optimized status quickly. To be more specific, in Fig. 9, since being implemented, the proposed approach takes 0.68 hour to regulate the given network to optimized status. This process can be accelerated if the control period is shorter than $1 \mathrm{~min}$, or a more aggressive problem segmentation mechanism in algorithm 1 is implemented. A shorter control period calls for more frequent broadcast and higher computational capability among users. A more aggressive segmentation leads to a more conservative control strategy when the proposed approach works under normal scenario. This is because without an explicit model to compute the optimal control policy, the proposed approach needs to either increase computational effort or employ greater steps in order to regulate the given LVDN from critical status back to optimized status faster. Nevertheless, with the proposed problem segmentation and feasible domain shrink in section III.C.(4), it is unlikely for the given LVDN to be in such a critical state unless there is complete control failure. Moreover, thanks to the decentralized decision making process in the proposed approach, controls are implemented independently among users, a complete control failure over the whole network is almost impossible either.

2) Communication malfunction: Communication malfunction includes two case studies. The first one is communication latency, in which all the users have a probability of $25 \%$ to encounter communication latency. A communication latency makes a user starts its control process later than others. In order to simplify the simulation, all the users are assumed to be able to solve three PDEs in one typical control period. Encountering communication latency is embodied by letting the user miss its time window for the first PDE solving. The communication latency results in asynchronous control among users and the latency is partially illustrated in Fig. 10 as an instance. Fig. 10 shows the communication status of 30 randomly chosen users in 30 minutes, from which one can see that at the beginning of every minute, some users suffer from communication latency randomly. The Yaxis is the nodal number, and the black bars indicate the communications. One can see for some users, their black bars start later than others and therefore leave some blanks in the control periods, which is the communication latency. The general average nodal voltage and evolvement of nodal

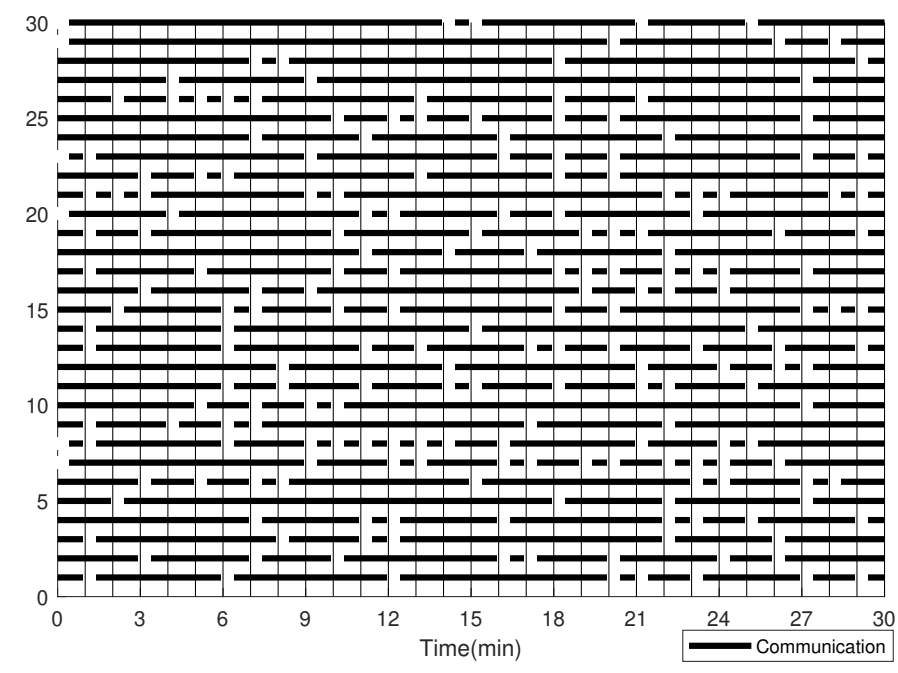

Fig. 10. Latency in communication 


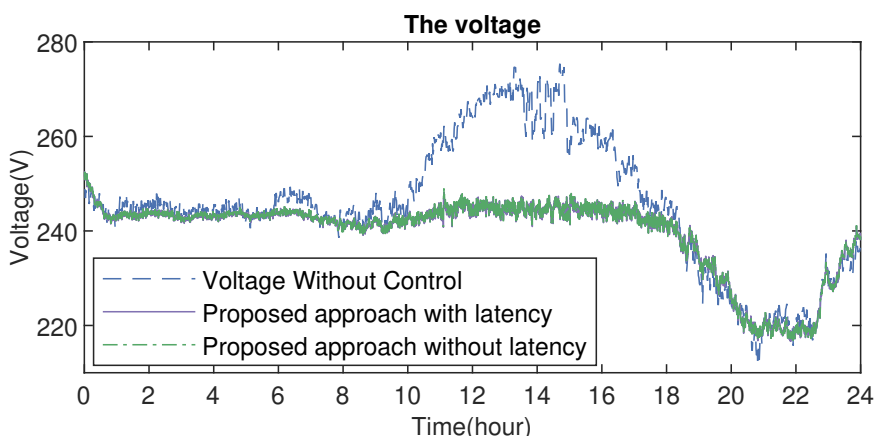

(a) The general average nodal voltage

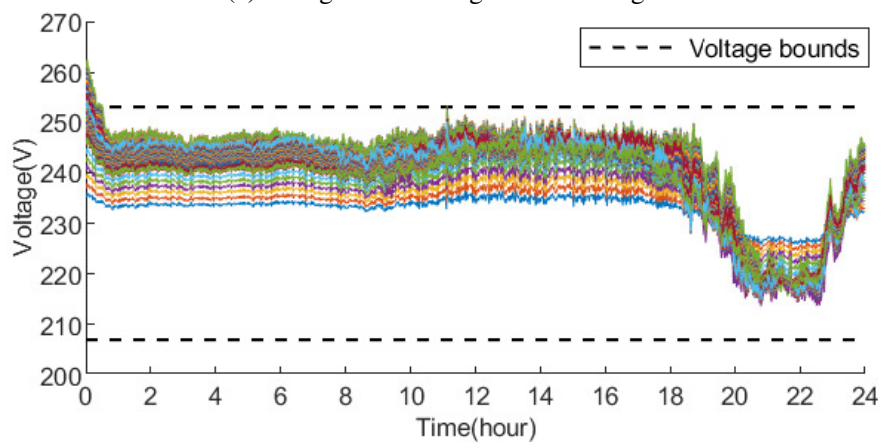

(b) Nodal voltages under normal communication

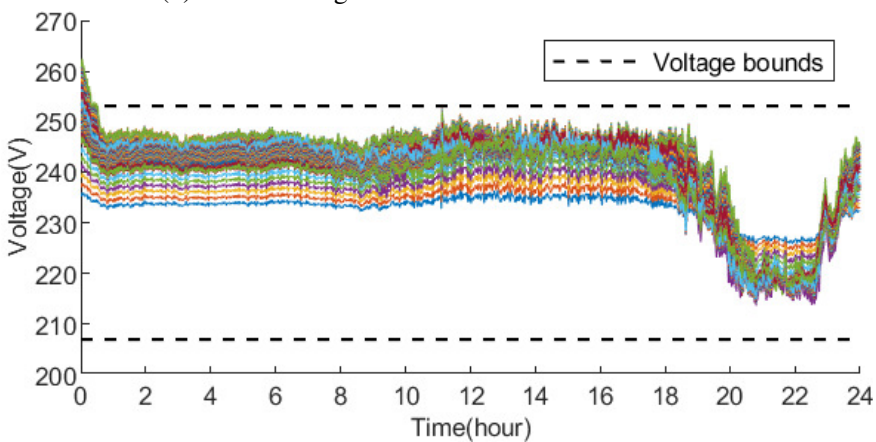

(c) Nodal voltages under communication with latency

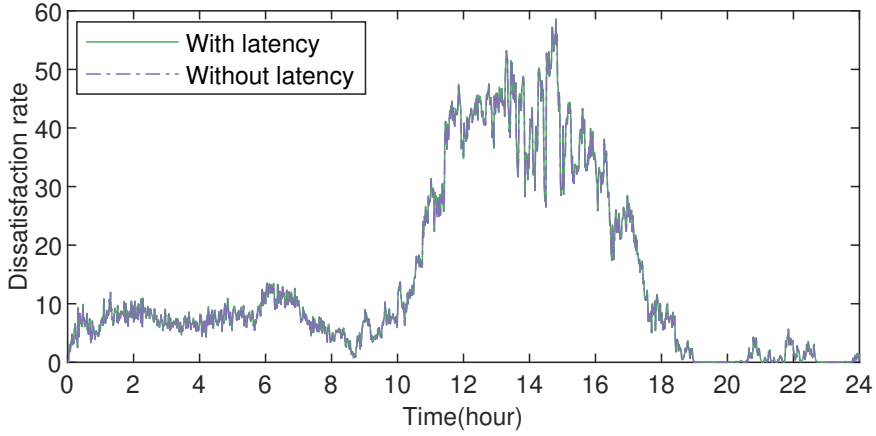

(d) Comparison on the dissatisfaction rates

Fig. 11. Special scenario of communication latency

voltages are shown in Fig. 11(a), 11(b) and 11(c) respectively. In the simulation, the power factor randomly changes between $[0.9,1]$ according to Belgian regulation, and $W=0.5$. The case with communication latency in Fig. 11(c) has the same power factor changes with the normal case in Fig. 11(b). Compared to the normal case without communication latency, the proposed algorithm gives almost same results with the latency. The comparison on dissatisfaction rates are given in Fig. 11(d), with the average $D_{t}$ of 14.1157 for the normal case

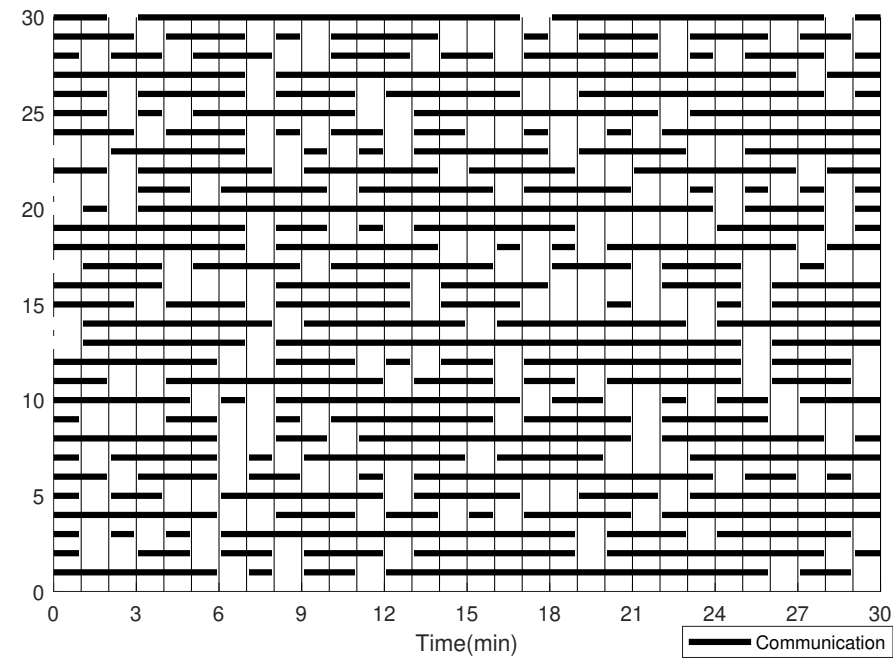

Fig. 12. packet loss in communication

and 14.1161 for the case with communication latency, which implies the almost same results. So far, it can be concluded that the proposed approach is able to deal with communication latency. The reason to it is that communication latency will result in asynchronous control among users, which is not a problem for the proposed control scheme, as the controls among users are inherently designed to be asynchronous in order to take different computational capability into account. The second special case study is communication malfunction. More specifically, the packet loss. Users are set to have a probability of $25 \%$ that cannot receive $\xi_{t}$ in a full control period. The embodiment is similar to the communication latency, if a user receives nothing after half of the preset typical control period, it will suppose a packet loss. Under this condition, it will take $\xi_{t}=0$ and start the control immediately. The user will solve its PDE only once to figure out a temporary control in the rest of the current control period. Similar to the previous special case, the communication status of packet loss is illustrated in Fig. 12, 30 randomly chosen users in 30 minutes are taken as an instance. Compared to Fig. 10, one can see the black bars are either full in a control period or completely blank, which indicates a packet loss. Consequently, the average nodal voltage and evolvement of nodal voltages are shown in Fig. 13(a), 13(b) and 13(c) respectively. Same to the case of communication latency, the power factor randomly changes between $[0.9,1]$ and $W=0.5$, and the case of packet loss in Fig. 13(c) has the same power factor changes with the normal case in Fig. 13(b). It is impossible for users to know it will be a packet loss instead of communication latency in advance, it only starts to realize it after receiving nothing for more than half of the set control period. Therefore PDE solving process is delayed in addition to assuming $\xi_{t}=0$. The comparison on dissatisfaction rate is given by Fig. 13(d), with the average $D_{t}$ of 14.1 for the normal case and 14.8 for the case with packet loss respectively. According to the results shown in Fig. 13, it is fair to claim that the proposed approach is able to deal with packet loss. Although there is a communication malfunction due to packet loss, as to the 


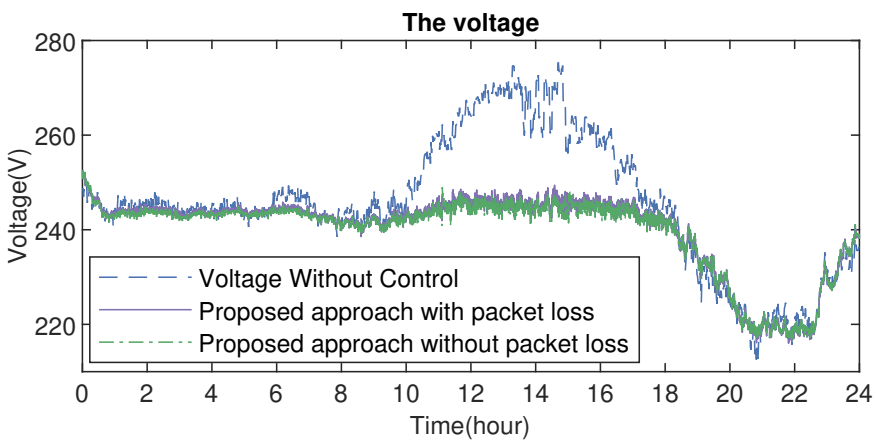

(a) The general average nodal voltage

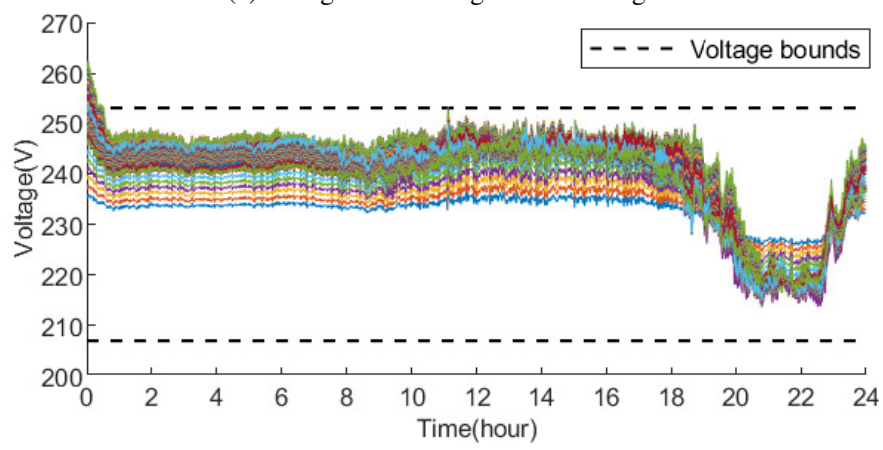

(b) Nodal voltages under normal communication

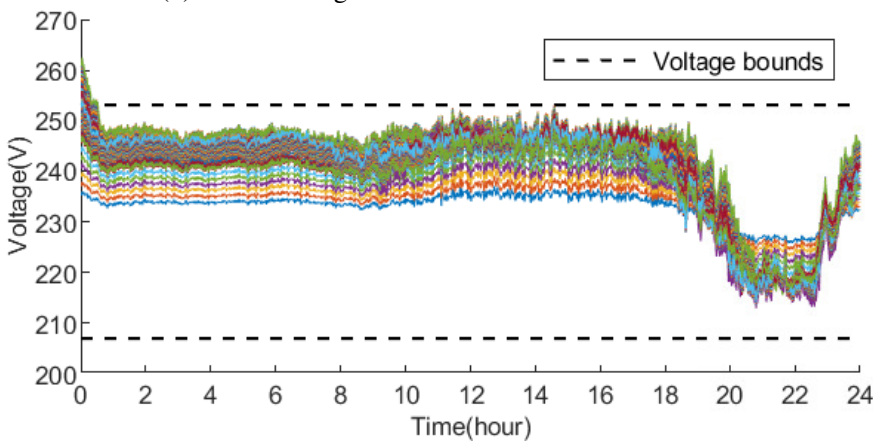

(c) Nodal voltages under communication with packet loss

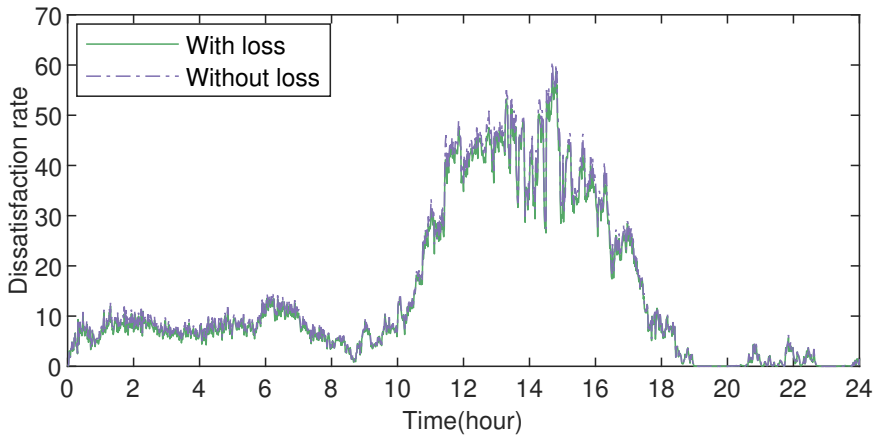

(d) Comparison on the dissatisfaction rates

Fig. 13. Special scenario of packet loss

affected user $i$, it is still able to keep updating $V_{t}^{i}$ to catch up with the global control scheme.

\section{CONClusion}

A distributed approach to seek approximate optimal control trajectory of users in LVDN with considering uncertainty is proposed. Compared to conventional centralized control, the proposed approach is model-free, demands much less communication efforts and is flexible to users with different computational capabilities. Moreover, as all the personal information is used locally, the concerns on user privacy are properly addressed. The case studies have proven that the proposed approach is able to deliver a good approximation to the theoretical optimal results in model-free and distributed manner. Moreover, the proposed approach is able to take uncertainties entered in different ways into account, which is proven in the case study as well.

Reactive power changes are considered as uncertainty in the paper, which fits the fact that most of the users in LVDN cannot control their active power and reactive power consumption individually. Nevertheless, it does not imply that the proposed approach is not able to consider active and reactive power separately. Actually, another PDE set similar to (28) can be established for reactive power, and the corresponding cost can be used as weight to indicate the share between active power and reactive power. Nevertheless, this will double the computation effort whereas it does not necessarily yield a significant improvement on performance.

From the case study one may have noticed that the parameter $W$ is set manually and same to every user. In fact, $W$ can be set and adjusted by a learning trial and error algorithm, which can measure and evaluate the uncertainty to give a proper $W$. Meanwhile, $W$ does not have to be same among different users, which is one of the advantages of the proposed approach. Since in the benchmark ACOPF, the margin can only be adjusted by modifying the cost function, which means it keeps same margin for all the users. Nevertheless, the uncertainty could be different if different perspective of the user is taken, the control can become more personalized by using non-homogeneous $W$, which yields an even better approximation to the optimum. This self-adaptive distributed control will be the future work.

The broadcast signal is not necessarily to be a rigid demand to the proposed approach. Depending on the communication topology, the broadcaster can be replaced by peer to peer communication, as long as it offers public information to keep the decentralized controls on same page. Alternatively, communication can be streamlined more if the Fokker-PlanckKolmogorov PDE or Mc-Kean Vlasov SDE is employed, then the public information can be offered with longer time interval, as it is just needed to caliber the controls, in case the error of the evaluated distribution becomes too large. Nevertheless, doing this will make the computation more intensive for users, to the best knowledge of the authors, it is a trade-off between communication and computation burdens. And the way to make a better trade-off can be a future work as well.

\section{REFERENCES}

[1] C. D'Adamo, S. Jupe, and C. Abbey, "Global survey on planning and operation of active distribution networks-update of cigre c6. 11 working group activities," in CIRED 2009-20th International Conference and Exhibition on Electricity Distribution-Part 1. IET, 2009, pp. 1-4.

[2] C. Le Floch, S. Bansal, C. J. Tomlin, S. J. Moura, and M. N. Zeilinger, "Plug-and-play model predictive control for load shaping and voltage control in smart grids," IEEE Transactions on Smart Grid, vol. 10, no. 3 , pp. 2334-2344, 2017.

[3] M. Yazdanian and A. Mehrizi-Sani, "Distributed control techniques in microgrids," IEEE Transactions on Smart Grid, vol. 5, no. 6, pp. 29012909, 2014. 
[4] K. E. Antoniadou-Plytaria, I. N. Kouveliotis-Lysikatos, P. S. Georgilakis, and N. D. Hatziargyriou, "Distributed and decentralized voltage control of smart distribution networks: Models, methods, and future research," IEEE Transactions on smart grid, vol. 8, no. 6, pp. 2999-3008, 2017.

[5] M. Falahi, K. Butler-Purry, and M. Ehsani, "Dynamic reactive power control of islanded microgrids," IEEE Transactions on Power Systems, vol. 28 , no. 4, pp. 3649-3657, 2013.

[6] R. Tang and S. Wang, "Model predictive control for thermal energy storage and thermal comfort optimization of building demand response in smart grids," Applied Energy, vol. 242, pp. 873-882, 2019.

[7] C. Lv, H. Yu, P. Li, C. Wang, X. Xu, S. Li, and J. Wu, "Model predictive control based robust scheduling of community integrated energy system with operational flexibility," Applied Energy, vol. 243, pp. 250-265, 2019.

[8] A. Nedic, A. Ozdaglar, and P. A. Parrilo, "Constrained consensus and optimization in multi-agent networks," IEEE Transactions on Automatic Control, vol. 55, no. 4, pp. 922-938, 2010.

[9] A. K. Mateska, V. Borozan, P. Krstevski, and R. Taleski, "Controllable load operation in microgrids using control scheme based on gossip algorithm," Applied Energy, vol. 210, pp. 1336-1346, 2018.

[10] J. Xu, H. Sun, and C. J. Dent, "Admm-based distributed opf problem meets stochastic communication delay," IEEE Transactions on Smart Grid, vol. 10, no. 5, pp. 5046-5056, 2018

[11] S. Magnússon, P. C. Weeraddana, and C. Fischione, "A distributed approach for the optimal power-flow problem based on admm and sequential convex approximations," IEEE Transactions on Control of Network Systems, vol. 2, no. 3, pp. 238-253, 2015.

[12] A. X. Sun, D. T. Phan, and S. Ghosh, "Fully decentralized ac optimal power flow algorithms," in 2013 IEEE Power \& Energy Society General Meeting. IEEE, 2013, pp. 1-5.

[13] H. Sira-Ramírez, A. Luviano-Juárez, M. Ramírez-Neria, and E. W. Zurita-Bustamante, Active disturbance rejection control of dynamic systems: a flatness based approach. Butterworth-Heinemann, 2018.

[14] J.-M. Lasry and P.-L. Lions, "Mean field games," Japanese journal of mathematics, vol. 2, no. 1, pp. 229-260, 2007.

[15] F. Bagagiolo and D. Bauso, "Mean-field games and dynamic demand management in power grids," Dynamic Games and Applications, vol. 4 no. 2, pp. 155-176, 2014.

[16] T. Hamidou, "Mean-field-type games in engineering," AIMS Journal, pp. 18-73, 2017.

[17] B. Wei and G. Deconinck, "Distributed model-free control in low voltage distribution networks: A mean field approach," in 2020 International Conference on Probabilistic Methods Applied to Power Systems (PMAPS). IEEE, 2020, pp. 1-6.

[18] D. Bauso, H. Tembine, and T. Başar, "Robust mean field games with application to production of an exhaustible resource," IFAC Proceedings Volumes, vol. 45, no. 13, pp. 454-459, 2012.

[19] R. Carmona, F. Delarue, and A. Lachapelle, "Control of mckeanvlasov dynamics versus mean field games," Mathematics and Financial Economics, vol. 7, no. 2, pp. 131-166, 2013.

[20] L. P. Kadanoff, Statistical physics: statics, dynamics and renormalization. World Scientific Publishing Company, 2000.

[21] R. Carmona, F. Delarue et al., Probabilistic Theory of Mean Field Games with Applications I-II. Springer, 2018.

[22] T. Başar, "Nash equilibria of risk-sensitive nonlinear stochastic differential games," Journal of optimization theory and applications, vol. 100, no. 3, pp. 479-498, 1999

[23] S. Zeadally, A.-S. K. Pathan, C. Alcaraz, and M. Badra, "Towards privacy protection in smart grid," Wireless personal communications, vol. 73, no. 1, pp. 23-50, 2013.

[24] Y. Liu, J. Li, L. Wu, and T. Ortmeyer, "Chordal relaxation based acopf for unbalanced distribution systems with DERs and voltage regulation devices," IEEE Transactions on Power Systems, vol. 33, no. 1, pp. 970984, 2018.

[25] H. Almasalma, S. Claeys, and G. Deconinck, "Peer-to-peer-based integrated grid voltage support function for smart photovoltaic inverters," Applied Energy, vol. 239, pp. 1037-1048, 2019.

[26] S. Pu, W. Shi, J. Xu, and A. Nedic, "Push-pull gradient methods for distributed optimization in networks," IEEE Transactions on Automatic Control, 2020.

[27] H. Laaksonen, P. Saari, and R. Komulainen, "Voltage and frequency control of inverter based weak lv network microgrid," in 2005 International Conference on Future Power Systems. IEEE, 2005, pp. 1-6.

[28] S. C. Dhulipala, R. V. A. Monteiro, R. F. da Silva Teixeira, C. Ruben, A. S. Bretas, and G. C. Guimarães, "Distributed model-predictive control strategy for distribution network volt/var control: A smart-building-based approach," IEEE Transactions on Industry Applications, vol. 55, no. 6, pp. 7041-7051, 2019

[29] P. Rutquist, C. Breitholtz, and T. Wik, "On the infinite time solution to state-constrained stochastic optimal control problems," Automatica, vol. 44, no. 7, pp. 1800-1805, 2008.

[30] P. Rutquist, T. Wik, and C. Breitholtz, "Solving the hamilton-jacobibellman equation for a stochastic system with state constraints," in 53rd IEEE Conference on Decision and Control. IEEE, 2014, pp. 18401845.

[31] B. Wei and G. Deconinck, "Distributed optimization in low voltage distribution networks via broadcast signals," Energies, vol. 13, no. 1, p. 43,2020

[32] C. S. Cheng and D. Shirmohammadi, "A three-phase power flow method for real-time distribution system analysis," IEEE Transactions on Power Systems, vol. 10, no. 2, pp. 671-679, 1995.

[33] C. C. David Fobes, "Threephasepowermodels," https://github.com/ lanl-ansi/ThreePhasePowerModels.j1.

[34] N. Etherden and M. H. Bollen, "Overload and overvoltage in low-voltage and medium-voltage networks due to renewable energy-some illustrative case studies," Electric Power Systems Research, vol. 114, pp. 39-48, 2014.

[35] P. Kadurek, J. Cobben, and W. Kling, "Overloading protection of future low voltage distribution networks," in 2011 IEEE Trondheim PowerTech. IEEE, 2011, pp. 1-6.

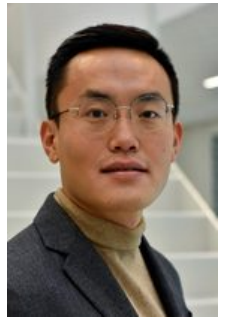

Boyuan Wei (Student Member, IEEE) received the B.Sc. degree in electrical engineering from Sichuan University, Chengdu, China in 2012, and the M.Sc. degree in electrical engineering from Southwest Jiaotong University, Chengdu, China in 2016. He is currently pursuing the $\mathrm{Ph} . \mathrm{D}$. degree in electrical engineering with KU Leuven, Leuven, Belgium. His main research interest is Distributed optimization in distribution networks and probabilistic methods applied to distribution networks.

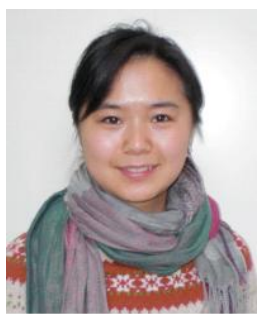

Zhifeng Qiu (Member, IEEE) received the M.S. degree in control engineering from Central South University, Changsha, China, and the Ph.D. degree in electrical engineering from KU Leuven, Leuven, Belgium, in 2004 and 2013, respectively. She is currently with Central South University. Her research interests include AI techonology and application in energy system, techno-economic problems in power systems as well as integration of renewable energy into network.

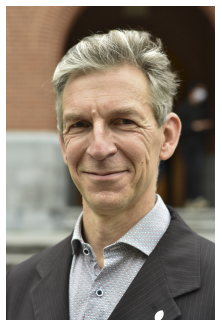

Geert Deconinck (Senior Member, IEEE) received the M.Sc. degree in electrical engineering and the $\mathrm{Ph} . \mathrm{D}$. degree in engineering science from KU Leuven, Belgium, in 1991 and 1996, respectively. He is currently a Full Professor with KU Leuven and with the EnergyVille research center. His research focuses on robust distributed coordination and control, specifically in the context of smart electric distribution networks. He is a Fellow of the Institute of Engineering and Technology and a Co-Chair of the IEEE Systems, Man, and Cybernetics Society Technical Committee on Infrastructure Systems and Services. 